\title{
DESENVOLVIMENTO IN VITRO DE HYPNEA PSEUDOMUSCIFORMIS, (GIGARTINALES, RHODOPHYTA) SOB CONDIÇÕES CONTROLADAS DE CULTURA
}

\author{
Daniela Dias Silva dos Reis'; Carlos Wallace do Nascimento Moura ${ }^{2}$ \\ 1. Bolsista PROBIC/UEFS, Graduanda em Ciências Biológicas, Universidade Estadual de Feira de Santana, e-mail: \\ dsr.reis@hotmail.com \\ 2. Orientador, Departamento de Ciências Biológicas, Universidade Estadual de Feira de Santana, e-mail: \\ wallace@uefs.br \\ PALAVRAS-CHAVE: Cultivo in vitro, Fisiologia, Algas.
}

\section{INTRODUÇÃO}

Hypnea pseudomusciformis Nauer, Cassano \& M.C. Oliveira é uma alga vermelha de grande interesse econômico que apresenta ampla distribuição no litoral brasileiro. Esta rodofícea, assim como demais representantes do gênero, apresentam talos ricos em polissacarídeos sulfatados, conhecidos como k-carragenana, de utilidade nas indústrias alimentícia, farmacêutica e cosmética (Nauer et. al. 2014).

Alguns estudos relacionados ao cultivo in vitro desta espécie foram realizados no Brasil, a fim de se analisar a interferência de fatores abióticos em seu crescimento. Bravin \& Yoneshigue-Valentin (2002), ao cultivarem a espécie em meio aerado e enriquecido com Provasoli's Enriched Seawater (PES), a uma temperatura de $25{ }^{\circ} \mathrm{C}$, verificaram que estes apresentaram um ótimo rendimento (biomassa de $17 \mathrm{~g}$ e taxa específica de crescimento de $20,79 \% \cdot$ dia $^{-1}$ ). Por sua vez, Martins \& Yokoya (2010) realizando um estudo para avaliar o efeito da disponibilidade do nitrogênio nos tipos morfológicos de diferentes cores de $H$. pseudomusciformis, constataram que estas cresceram melhor em meio Von Stoch (VS) que em meio artificial ASP 12-NTA.

Diante da ausência de estudos fisiológicos de rodofíceas marinhas no Estado, o presente trabalho visou o cultivo in vitro de $H$. pseudomusciformis a partir do desenvolvimento de fragmentos apicais submetidos a diferentes meios, temperatura e aeração.

\section{MATERIAL E MÉTODOS}

Para a realização deste trabalho, exemplares de linhagem marrom de $H$. pseudomusciformis foram coletados em ambiente recifal na Praia da Penha, município de Vera Cruz no litoral baiano.

Fragmentos apicais de $15 \mathrm{~mm}$ foram removidos desses talos e submetidos a cultivo in vitro, a fim de se analisar a influência de fatores abióticos em seu crescimento, tais como temperatura $\left(25\right.$ e $\left.28^{\circ} \mathrm{C}\right)$, presença ou ausência de aeração, e meio enriquecido (Provasoli e Von Stoch).

O experimento foi feito em duas etapas, cada qual com 40 dias de duração, com troca periódica do meio de cultivo e medições de biomassa realizadas a cada 10 dias. Três fragmentos apicais com gavinhas foram inseridos em frascos "baby food" contendo $100 \mathrm{ml}$ de meios de cultivo (salinidade 34 UPS e $\mathrm{pH} 8,2$ ).

Para cada tratamento (água do mar, Provasoli e Von Stoch), cinco frascos correspondentes foram submetidos à aeração a partir de mangueiras acopladas a uma bomba de aquário, e cinco frascos não foram submetidos à aeração. Os experimentos foram submetidos a um fotoperíodo de $12: 12 \mathrm{~h}$ (luz : escuro) e irradiância de $30 \pm 5 \mu$ mol fótons.m ${ }^{-}$ ${ }^{2} . \mathrm{s}^{-1}$. Na primeira etapa, realizada durante os meses de janeiro e fevereiro, os frascos foram cultivados a temperatura de $25{ }^{\circ} \mathrm{C}$. A segunda etapa foi realizada utilizando-se a temperatura de $28{ }^{\circ} \mathrm{C}$ e ocorreu durante os meses de abril e maio.

Os dados obtidos foram submetidos a analise de variância, com auxílio do software estatístico R. 


\section{RESULTADOS E DISCUSSÃO}

A partir da análise de variância pode-se constatar que a $25{ }^{\circ} \mathrm{C}$, os fatores que influenciaram significativamente o crescimento dos fragmentos de $H$. pseudomusciformis foram os meios de cultivo $\left(\chi^{2}=68,20, \mathrm{gl}=1, \mathrm{p}<0,001\right)$ e os meios de cultivo combinados com o fator aeração $\left(\chi^{2}=7,60, \mathrm{gl}=2, \mathrm{p}=0,02\right.$; Fig. 1$)$. Enquanto a $28{ }^{\circ} \mathrm{C}$, apenas os meios de cultivo tiveram influência significativa sobre o crescimento $\left(\chi^{2}=75,4, \mathrm{gl}=1, \mathrm{p}<0,001\right)$. Sob esta temperatura, os talos adquiriram discreta biomassa (Fig. 2) na presença de aeração (CA), embora não significativa $\left(\chi^{2}=0,9, \mathrm{gl}=2, \mathrm{p}>0,05\right)$. Observou-se que, tanto nos meios aerados quanto nos não aerados, os maiores valores de biomassa foram encontrados no meio VS, que, em geral, a partir do $20^{\circ}$ dia, superaram os valores de biomassa dos fragmentos cultivados em meio PES (Tab. 1).

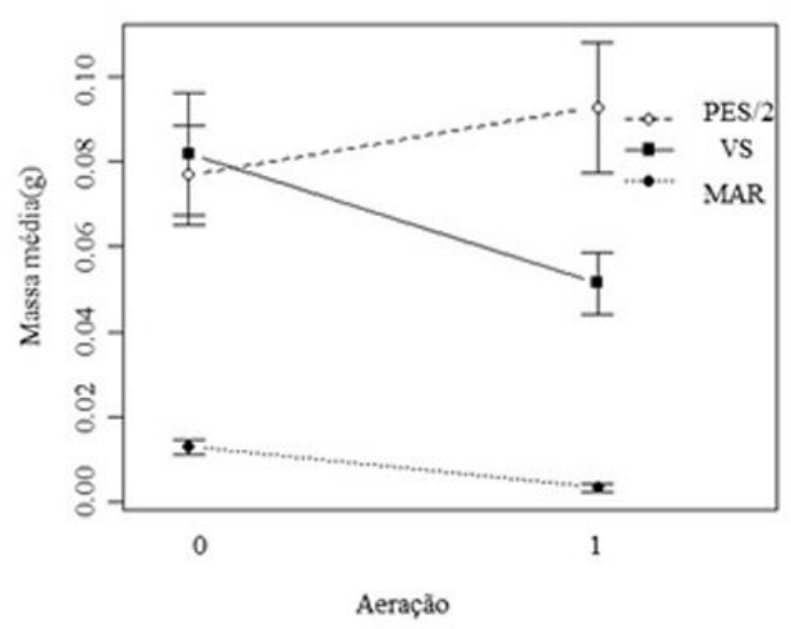

Figura 1. Crescimento médio (g) dos talos de $H$. pseudomusciformis cultivados em meio Provasoli (PES/2), Von Stosch (VS) e água do mar (MAR) a $25^{\circ} \mathrm{C}$, na ausência (0) e presença (1) de aeração.

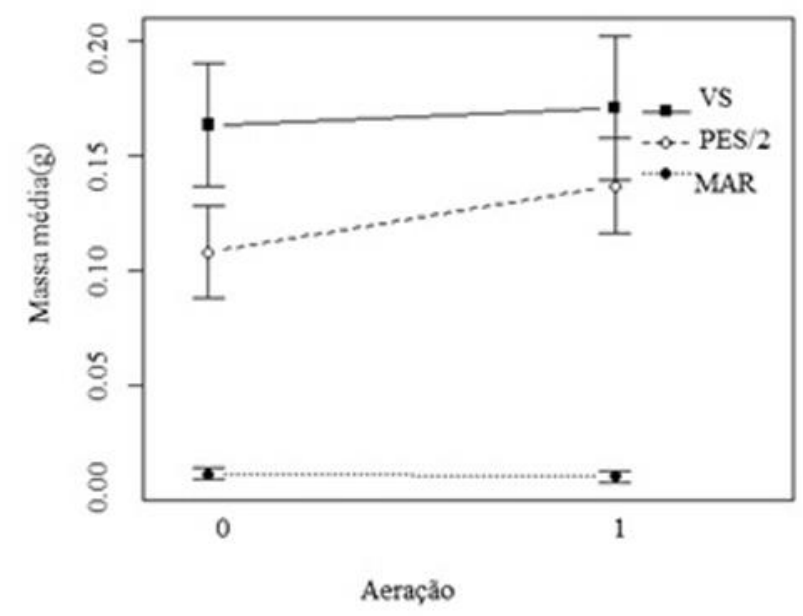

Figura 2. Crescimento médio (g) dos talos de $H$. pseudomusciformis cultivados em meio Provasoli (PES/2), Von Stosch (VS) e água do mar (MAR) a $28^{\circ} \mathrm{C}$, na ausência (0) e presença (1) de aeração. 
Tabela 1. Média das taxas de crescimento $\left(\% \cdot \mathrm{dia}^{-1}, \mathrm{n}=5\right)$ dos talos de Hypnea pseudomusciformis cultivados a $25^{\circ} \mathrm{C}$ e $28^{\circ} \mathrm{C}$, com aeração (CA) e sem aeração (SA), em meio Provasoli (PES/2), Von Stosch (VS) e água do mar (MAR).

\begin{tabular}{|c|c|c|c|c|c|c|c|c|c|c|c|c|}
\hline \multirow{4}{*}{ Dias } & \multicolumn{12}{|c|}{ Taxas de Crescimento } \\
\hline & \multicolumn{6}{|c|}{$25^{\circ} \mathrm{C}$} & \multicolumn{6}{|c|}{$28^{\circ} \mathrm{C}$} \\
\hline & \multicolumn{3}{|c|}{ CA } & \multicolumn{3}{|c|}{ SA } & \multicolumn{3}{|c|}{ CA } & \multicolumn{3}{|c|}{ SA } \\
\hline & PES/2 & VS & MAR & PES/2 & VS & MAR & PES/2 & VS & MAR & PES/2 & VS & MAR \\
\hline 10 & 12,71 & 15,04 & 1,20 & 12,99 & 12,53 & 3,79 & 13,98 & 13,88 & 5,20 & 11,34 & 11,56 & 5,42 \\
\hline 20 & 3,70 & $-2,14$ & - & 5,38 & 7,08 & 2,55 & 8,57 & 9,03 & $-0,56$ & 8,75 & 12,83 & 2,27 \\
\hline 30 & 4,16 & $-0,47$ & - & 4,04 & 3,57 & 1,31 & 3,06 & 4,67 & - & 1,15 & 4,16 & - \\
\hline 40 & 1,48 & 7,77 & - & 4,22 & 5,08 & - & 2,91 & 2,97 & - & 2,31 & 4,21 & - \\
\hline
\end{tabular}

O meio enriquecido é um fator de relevância para cultivos em laboratório. Outros estudos como aquele realizado por Bravin \& Yoneshigue-Valentin (2002), mostrou que o enriquecimento foi o fator mais importante dentre outras condições citadas. Martin \& Yokoya (2010), verificaram que espécimes verde-claros, verde-escuros e marrons de $H$. musciformis (= H. pseudomusciformis) obtiveram maior biomassa quando cultivados em meio VS que em meio ASP 12-NTA. Yokoya et al. (2006) notaram que os exemplares da mesma espécie submetidos a diferentes valores de irradiância cresceram mais na faixa de temperatura entre 20-25 ${ }^{\circ} \mathrm{C}$ para os fotoperídos de 10:14 h e 14: $10 \mathrm{~h}$ (claro: escuro) que na temperatura de $30^{\circ} \mathrm{C}$.

Devido à ampla distribuição de $H$. pseudomusciformis no litoral brasileiro é de se esperar que esta espécie seja adaptada a diferentes valores de temperaturas. Bravin \& Yoneshigue-Valentin (2002) concluíram que a temperatura foi o segundo fator de relevância para o crescimento de $H$. musciformis ( $=H$. pseudomusciformis).

No presente experimento, as algas submetidas à temperatura de $28{ }^{\circ} \mathrm{C}$ obtiveram maior ganho de biomassa. É importante ressaltar que esta temperatura está mais próxima daquela mensurada em local de coleta (cerca de $27,5^{\circ} \mathrm{C}$ ).

Nas duas etapas experimentais, observou-se que os talos cultivados em meios nãoenriquecidos não foram capazes de sobreviver durante os 40 dias de observações. Em cultivos aerados, talos morreram com 20 dias de experimento e em cultivos não aerados, a morte ocorreu a partir do $30^{\circ}$ dia. Para os demais meios, notou-se um progressivo clareamento dos fragmentos a partir do 200 dia de experimento, com tendência a permanecer um pouco mais escuros em meios VS que em meios PES.

\section{CONSIDERAÇÕES FINAIS}

Os resultados mostram que os meios de enriquecimento tiveram grande importância no crescimento desta espécie in vitro. Talos que cresceram em meio não enriquecido, ou seja, cultivadas apenas em água do mar, não sobreviveram até o $40^{\circ}$ dia de cada etapa do experimento. Além disso, as algas apresentaram maiores ganhos de biomassa para a temperatura de $28^{\circ} \mathrm{C}$.

\section{REFERÊNCIAS}

BRAVIN I.C., YONESHIGUE-VALENTIN Y. 2002. Influência de fatores ambientais sobre o crescimento in vitro de Hypnea musciformis (Wulfen) Lamouroux (Rhodophyta). Revista Brasileira de Botânica. 25(4):469-474. 
BRAVIN I.C., YONESHIGUE-VALENTIN, Y.,YOKOYA N.S. 2006. Formação de calos e regeneração de seguimentos apicais de Hypnea musciformis (Wulfen) Lamouroux (Gigartinales, Rodophyta): obtenção de culturas axênicas e efeito da concentração do ágar. Revista Brasil. Bot. 29:175-182.

MARTIN A.P., YOKOYA N.S. 2010. Intraespecific variations in colour morphs of Hypnea musciformis (Rodophyta) in relation to nitrogen availability. Hoehnea. 37(3):601-615.

NAUER F., CASSANO V, OLIVEIRA M.C. 2014. Description of Hypnea pseudomusciformis sp. nov., a new species based on molecular and morphological analyses, in the context of the H. musciformis complex (Gigartinales, Rodophyta). Journal of Applied Phycology. DOI 10.1007/s 10811-014-0488-y.

$\mathrm{R}$ : A language and environment for statistical computing. R Foundation for Statistical Computing, Vienna, Austria. ISBN 3-900051-07-0, URL http://www.R-project.org/. 